Proc. of the 15th Int. Workshop on Slow Positron Beam Techniques and Applications, Prague, September 2-6, 2019

\title{
Calculation of Positron States and Annihilation Parameters in Gamma and Amorphous $\mathrm{Al}_{2} \mathrm{O}_{3}$
}

\author{
S. IshiBASHI ${ }^{a, *}$ AND A. UEDONO ${ }^{b}$ \\ ${ }^{a}$ Research Center for Computational Design of Advanced Functional Materials (CD-FMat), \\ National Institute of Advanced Industrial Science and Technology (AIST), Tsukuba, Ibaraki 305-8568, Japan \\ ${ }^{b}$ Division of Applied Physics, Faculty of Pure and Applied Science, University of Tsukuba, Tsukuba, \\ Ibaraki 305-8573, Japan
}

\begin{abstract}
We calculate positron states and annihilation parameters theoretically in gamma and amorphous $\mathrm{Al}_{2} \mathrm{O}_{3}$ and compared with those in alpha $\mathrm{Al}_{2} \mathrm{O}_{3}$. They show systematic variations and are investigated in terms of relationships with microscopic structures and densities. Good correlations of the positron annihilation parameters with the density are observed.
\end{abstract}

DOI: 10.12693/APhysPolA.137.231

PACS/topics: 71.60.+z, 78.70.Bj, 71.55.Jv

\section{Introduction}

Gallium nitride and related compounds attract much attention because of their applicability not only in lightemitting devices but also in power devices. The quality of gate insulator material plays a crucial role for the device performance and $\mathrm{Al}_{2} \mathrm{O}_{3}$ is a promising candidate. It is thought that deposited $\mathrm{Al}_{2} \mathrm{O}_{3}$ layers have various structures depending on the preparation method and condition. The positron annihilation spectroscopy is expected to be applied in evaluating their qualities $[1,2]$. Recently, we applied slow positron beams in characterizing gate-stack $\mathrm{Al}_{2} \mathrm{O}_{3}$ layers [3].

In the present study, we construct appropriate structural models of gamma $(\gamma-)$ and amorphous (a-) $\mathrm{Al}_{2} \mathrm{O}_{3}$, which are often observed in real insulator layers. Positron states and annihilation parameters (the positron annihilation Doppler broadening $S$ and $W$ parameters as well as positron lifetime $\tau$ ) therein are calculated and compared with those in alpha $(\alpha-) \mathrm{Al}_{2} \mathrm{O}_{3}$.

\section{Computational details}

Structural models of $\gamma-\mathrm{Al}_{2} \mathrm{O}_{3}$ are prepared by reference to [4]. The $\gamma-\mathrm{Al}_{2} \mathrm{O}_{3}$ crystal has a defect cubic spinel structure. To obtain a stoichiometric $\mathrm{Al}_{2} \mathrm{O}_{3}$, three cubic cells are needed. An equivalent hexagonal supercell is used in this study. By removing $8 \mathrm{Al}$ atoms, a stoichiometric $\mathrm{Al}_{64} \mathrm{O}_{96}$ structure is constructed. There are two different crystallographic $\mathrm{Al}$ sites in the cubic spinel structure, the octahedral $(O)$ and tetrahedral $(T)$ sites. The theoretical work by Streitz and Mintmire [5] indicated that, although it is energetically favorable when all vacant $\mathrm{Al}$ sites are located at $O$ sites, the increase in energy when $T$ sites are occupied with a modest fraction

*corresponding author; e-mail: shoji.ishibashi@aist.go.jp is small. Therefore, we construct $16+16$ different structural models by randomly removing $8 \mathrm{Al}$ atoms from the $O$ sites only or from both the $O$ and $T$ sites.

Structural models of a- $\mathrm{Al}_{2} \mathrm{O}_{3}$ are prepared as follows. $64 \mathrm{Al}$ and $96 \mathrm{O}$ atoms are randomly distributed in cubic cells, whose lattice parameters are determined so that the density is $3.0,3.3$, or $3.5 \mathrm{~g} / \mathrm{cm}^{3}$. First-principles molecular dynamics (MD) calculations are performed for each system using the Langevin thermostat as follows: (1) $T=3000 \mathrm{~K}, \Delta t=0.2 \mathrm{fs}, \zeta=10 \mathrm{ps}^{-1}$ for 0-0.5 ps, (2) $T=3000 \mathrm{~K}, \Delta t=0.5 \mathrm{fs}, \zeta=10 \mathrm{ps}^{-1}$ for $0.5-2.0 \mathrm{ps}$, (3) $T=3000 \mathrm{~K}, \Delta t=1.0 \mathrm{fs}, \zeta=0.01 \mathrm{ps}^{-1}$ for 2.0-10.0 ps, (4) $T=1500 \mathrm{~K}, \Delta t=1.0 \mathrm{fs}, \zeta=1 \mathrm{ps}^{-1}$ for $10.0-14.0 \mathrm{ps}$, and (5) $T=1500 \mathrm{~K}, \Delta t=1.0 \mathrm{fs}$, $\zeta=0.01 \mathrm{ps}^{-1}$ for $14.0-22.0 \mathrm{ps}$, where $T, \Delta t$, and $\zeta$ represent a temperature, a time interval, and a friction parameter, respectively. From MD runs at $1500 \mathrm{~K}$ for each density, snapshots at every 1000 steps (1 ps) are extracted and the atomic positions therein are optimized. The obtained structures are reasonable when compared with previous studies [6, 7].

Structural models of defect-free $\alpha-\mathrm{Al}_{2} \mathrm{O}_{3}$ and a system containing $V_{5}$, where two $\mathrm{Al}$ sites and three $\mathrm{O}$ sites are vacant, are also prepared using an orthorhombic supercell equivalent to $2 \times 2 \times 1$ hexagonal unit cells.

On these structural models, positron states and annihilation parameters $S, W$, and $\tau$ are calculated with our computational code QMAS [8]. The $S$ and $W$ parameters are evaluated using spherically averaged momentum densities. As for the enhancement factor, we use the form $\gamma=1+\left(\gamma_{\mathrm{BN}}-1\right)\left(1-1 / \varepsilon_{\infty}\right)$, where $\gamma_{\mathrm{BN}}$ is the BorońskiNieminen enhancement factor [9] and $\varepsilon_{\infty}$ is the highfrequency dielectric constant. This form is slightly different from the original semiconductor model [10]. It follows the form in the positron generalized-gradient approximation [11] and is consistent with the scaled positronelectron correlation energy [10]. Further details are described in our recent paper [12]. $\varepsilon_{\infty}$ is set to be 3.0 in the present study. 


\section{Results and discussion}

Figure 1 represents the calculated positron annihilation parameters in 16 [octahedral sites only $(O$ only)] +16 [octahedral and tetrahedral sites $(O+T)$ ] structural models of $\gamma-\mathrm{Al}_{2} \mathrm{O}_{3}$. Some of the positron-lifetime results have been already reported in Ref. [3]. Reflecting variations in their structures, the resultant $S, W$, and $\tau$ show finite distributions. The average $(S, W, \tau)$ values are $(0.4376,0.01533,239.0 \mathrm{ps})$ for the " $O$ only" case and $(0.4396,0.1516,244.9 \mathrm{ps})$ for the " $O+T$ " case, respectively. Although the differences between the two cases are not large, it seems slightly more likely that vacant sites are closely located from each other in the " $O+T$ " case.

In Fig. 2, the calculated positron annihilation parameters for structures optimized from snapshots in MD runs at $1500 \mathrm{~K}$ are shown as a function of time. Although fluctuations exist, they show clear dependencies on the density. The calculated $\tau$ values for a- $\mathrm{Al}_{2} \mathrm{O}_{3}$ of the 3.3 or $3.5 \mathrm{~g} / \mathrm{cm}^{3}$ cases as well as those in $\gamma-\mathrm{Al}_{2} \mathrm{O}_{3}$ are in good agreement with the values for the shortest component of the experimentally obtained lifetime spectra [3]. The calculated positron annihilation parameters for the defectfree (DF) state and $V_{5}$ state in $\alpha-\mathrm{Al}_{2} \mathrm{O}_{3}$ are listed in Table I. Although the present $\tau$ value for the DF state is slightly longer than the experimental value of 143 ps [13], it is still in a reasonable range.

In Fig. 3, the calculated $S$ and $\tau$ values for various structures are plotted for comparison. Several corresponding positron density distributions are also shown. Although there is a small but significant separation between $\gamma$ - and a- $\mathrm{Al}_{2} \mathrm{O}_{3}$, all the points are on roughly the same line, showing a clear correlation between $S$ and $\tau$. On the other hand, a good negative correlation exists between $S$ and $W$. As mentioned above, finite distributions
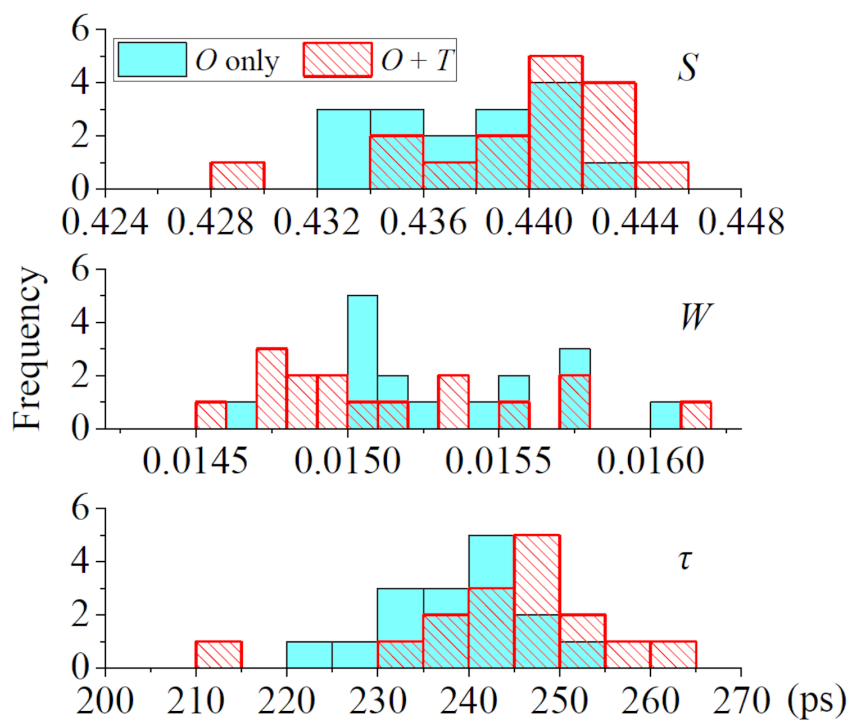

Fig. 1. Frequency distributions of calculated positron annihilation parameters in 16 (octahedral sites only $(O$ only) $)+16$ (octahedral and tetrahedral sites $(O+T)$ ) structural models of $\gamma-\mathrm{Al}_{2} \mathrm{O}_{3}$.

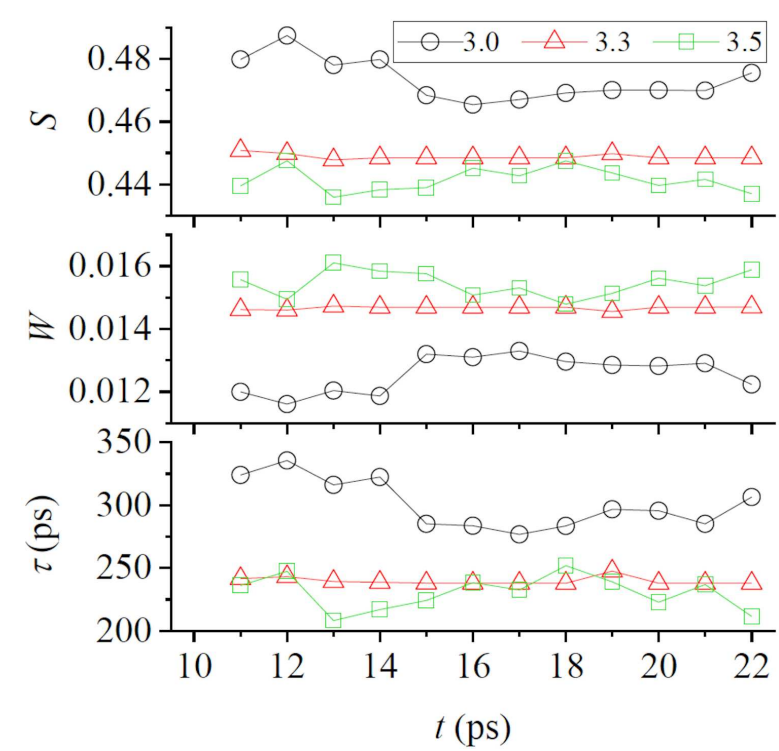

Fig. 2. Calculated positron annihilation parameters for structures optimized from snapshots in MD runs at $1500 \mathrm{~K}$. Numbers in legends represent density values in $\mathrm{g} / \mathrm{cm}^{3}$.

TABLE I

Calculated positron annihilation parameters for the defect-free (DF) state and $V_{5}$ state in $\alpha-\mathrm{Al}_{2} \mathrm{O}_{3}$.

\begin{tabular}{c|c|c|c}
\hline \hline & $S$ & $W$ & $\tau[\mathrm{ps}]$ \\
\hline $\mathrm{DF}$ & 0.411 & 0.0188 & 158.9 \\
$V_{5}$ & 0.462 & 0.0139 & 287.0
\end{tabular}

of these parameters are observed for $\gamma$ - and $\mathrm{a}-\mathrm{Al}_{2} \mathrm{O}_{3}$. To make further quantitative statistical analyses, more data are required. The positron density distribution show large variations from a completely delocalized state to a localized state trapped at an open space.

In Fig. 4, the calculated $S, W$, and $\tau$ are represented as a function of the density $\rho$. The lower bounds of $S$ and $\tau$ as well as the upper bounds of $W$ at respective $\rho$ values for a- and $\alpha-\mathrm{Al}_{2} \mathrm{O}_{3}$ look to be on the same straight line. If an arbitrary part of the structure is a good representative of the whole structure, it is quite reasonable that the positron annihilation parameters $S, W$, and $\tau$ show clear relationships with $\rho$. The defect free state of $\alpha-\mathrm{Al}_{2} \mathrm{O}_{3}$ and some of a- $\mathrm{Al}_{2} \mathrm{O}_{3}$ structures are thought to fulfill such a condition. Positron distributions therein are delocalized, and the resultant positron parameters are expected to bring average information of the system. The $V_{5}$ case of $\alpha-\mathrm{Al}_{2} \mathrm{O}_{3}$ is completely opposite. The positron distribution is strongly localized, and only information in the vicinity of the vacancy is brought. Therefore, the results for $V_{5}$ in Fig. 4 are far off the straight lines. As mentioned above, the structure of $\gamma-\mathrm{Al}_{2} \mathrm{O}_{3}$ is characterized by the presence of the cation-site vacancies, although the degree of localization is weaker that that for the $V_{5}$ case in $\alpha-\mathrm{Al}_{2} \mathrm{O}_{3}$. Then, the lower or upper bounds for $\gamma-\mathrm{Al}_{2} \mathrm{O}_{3}$ are slightly off the lines. 


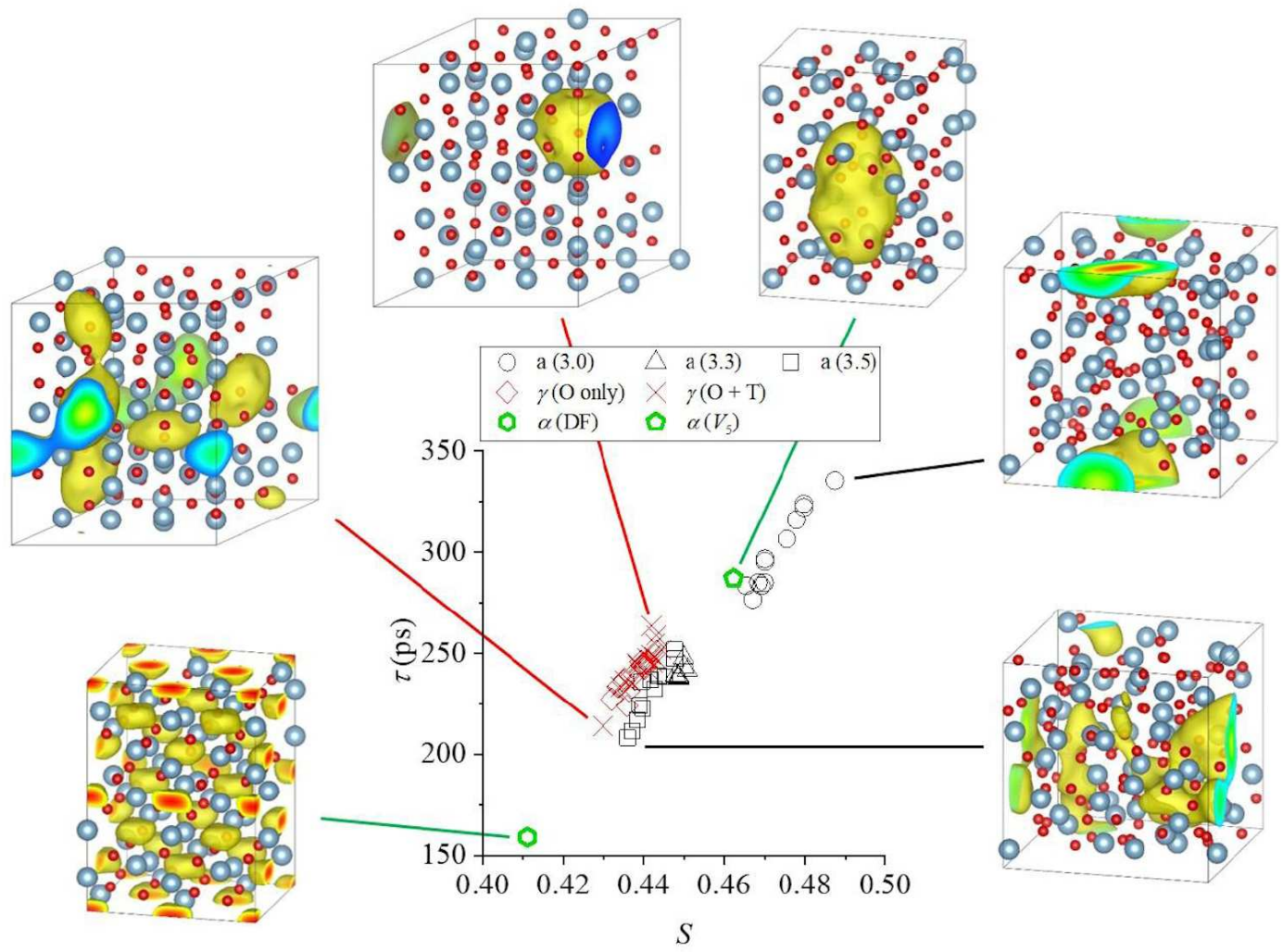

Fig. 3. $S$ and $\tau$ in various structures of $\mathrm{Al}_{2} \mathrm{O}_{3}$ and several corresponding positron density distributions. VESTA [14] was used in drawing a part of figure.

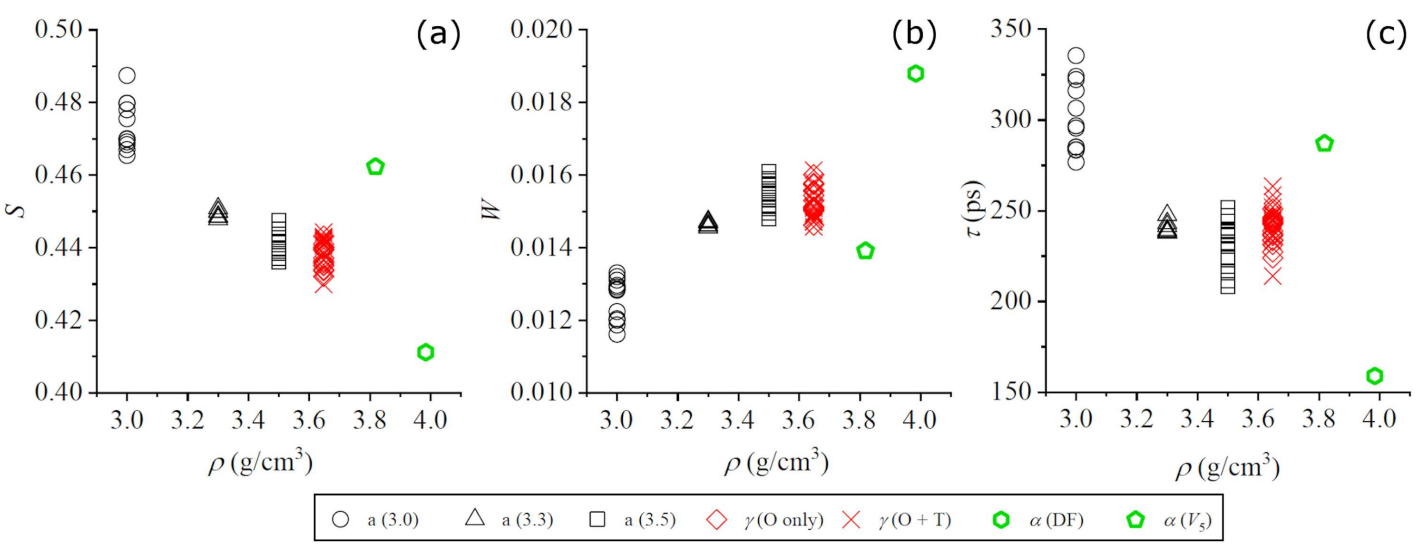

Fig. 4. Calculated $S(\mathrm{a}), W(\mathrm{~b})$, and $\tau(\mathrm{c})$ as a function of $\rho$.

\section{Summary}

We have successfully modeled reasonable structures of $\gamma$ - and $\mathrm{a}-\mathrm{Al}_{2} \mathrm{O}_{3}$. The positron states and annihilation parameters therein are calculated and compared with those in $\alpha-\mathrm{Al}_{2} \mathrm{O}_{3}$. As for the $S, W$, and $\tau$ values in $\gamma-\mathrm{Al}_{2} \mathrm{O}_{3}$ and $\mathrm{a}-\mathrm{Al}_{2} \mathrm{O}_{3}$, finite distributions are observed reflecting variations in their structures. The $\tau$ values in $\gamma-\mathrm{Al}_{2} \mathrm{O}_{3}$ and a- $\mathrm{Al}_{2} \mathrm{O}_{3}$ with the density of 3.3 or $3.5 \mathrm{~g} / \mathrm{cm}^{3}$ are in good agreement with the experimental results. The $S$,
$W$, and $\tau$ values in various phases of $\mathrm{Al}_{2} \mathrm{O}_{3}$ show good correlations with one another. They also show characteristic relationships with the density, which can be applied in characterizing $\mathrm{Al}_{2} \mathrm{O}_{3}$ insulator layers by the positron annihilation technique.

Since local structures of $\gamma-\mathrm{Al}_{2} \mathrm{O}_{3}$ and $\mathrm{a}-\mathrm{Al}_{2} \mathrm{O}_{3}$ have an infinite number of possible configurations, it is important to accumulate further results of calculations. Effects of dopants and/or impurities as well as those of thermal history should be investigated as future issues. 


\section{Acknowledgments}

This work was supported by the MEXT "Program for research and development of next-generation semiconductor to realize energy-saving society".

\section{References}

[1] R. Krause-Rehberg, H.S. Leipner, Positron Annihilation in Semiconductors, Springer, Berlin 1999.

[2] F. Tuomisto, I. Makkonen, Rev. Mod. Phys. 85, 1583 (2013).

[3] A. Uedono, T. Nabatame, W. Egger, T. Koschine, C. Hugenschmidt, M. Dickmann, M. Sumiya, S. Ishibashi, J. Appl. Phys. 123, 155302 (2018).

[4] E. Menéndez-Proupin, G. Gutiérrez, Phys. Rev. B 72, 035116 (2005).

[5] F.H. Streitz, J.W. Mintmire, Phys. Rev. B 60, 773 (1999).
[6] G. Gutiérrez, A.B. Belonoshko, R. Ahuja, B. Johansson, Phys. Rev. E 61, 2723 (2000).

[7] G. Gutiérrez, B. Johansson, Phys. Rev. B 65, 104202 (2002).

[8] S. Ishibashi, T. Tamura, S. Tanaka, M. Kohyama, K. Terakura, Phys. Rev. B 76, 153310 (2007).

[9] E. Boroński, R.M. Nieminen, Phys. Rev. B 34, 3820 (1986).

[10] M.J. Puska, S. Mäkinen, M. Manninen, R.M. Nieminen, Phys. Rev. B 39, 7666 (1989).

[11] B. Barbiellini, M.J. Puska, T. Torsti, R.M. Nieminen, Phys. Rev. B 517341 (1995).

[12] S. Ishibashi, A. Uedono, H. Kino, T. Miyake, K. Terakura, J. Phys. Condens. Matter 31, 475401 (2019).

[13] A. Uedono, K. Ikeuchi, K. Yamabe, J. Appl. Phys. 98, 023506 (2005).

[14] K. Momma and F. Izumi, J. Appl. Crystallogr. 44, $1272(2011)$ 